\title{
Er-doped Superfluorescent Fiber Source with Thermally Stable Mean Wavelength
}

\author{
Hee Gap Park* and Seung Chul Yun \\ Department of Physics, Chonbuk National University, Jeonju 561-756, Korea \\ Young Jun Jin \\ ISR RED Center, LIG Nex1 Co. Ltd., Youngin 148-1, Korea
}

(Received April 21, 2009 : revised June 5, 2009 : accepted June 11, 2009)

\begin{abstract}
An Er-doped superfluorescent fiber source is designed and constructed with the double-pass forward configuration, which aims at high stability of its mean wavelength against temperature variation. As a result, thermal stability of mean wavelength better than $1 \mathrm{ppm}$ is obtained against the temperature variation of $\pm 5{ }^{\circ} \mathrm{C}$ around the optimum operating temperature. The optimum operating temperature can be tuned with the Er-doped fiber length and the pump power.
\end{abstract}

Keywords: Er-doped fiber, Optical fiber sensor, Optical fiber, Superfluorescent source

OCIS codes : (060.2370) Fiber optics sensors; (060.2410) Fibers, erbium; (060.2800) Gyroscopes

\section{INTRODUCTION}

Broadband Er-doped fiber (EDF) sources have been studied extensively for their application as light sources in optical fiber sensors, especially in the fiber-optic gyroscope (FOG) [1-4]. For the implementation of sources for inertial grade FOGs, stabilization of the mean wavelength has been one of the critical issues [5]. This is because the scale factor between the rotation rate and the measured Sagnac phase shift is directly related to the mean wavelength of the source [6].

Many factors can affect the mean-wavelength stability of the EDF sources. Among them, polarization-related effects and temperature dependences are the most important remaining issues of instabilities. Polarization effects arise from polarization-dependent gain (PDG) in the EDF [7], which can induce differences in mean wavelength between two eigenpolarizations of the source output, causing the drift of the mean wavelength when perturbations are involved. The source configuration of 'double-pass forward (DPF)' can be a good candidate to solve these instability problems. The reason is that most of polarization effects can be eliminated by incorporation of a Faraday-rotating mirror (FRM) in this

*Corresponding author: hgpark@chonbuk.ac.kr configuration $[8,9]$. Regarding temperature effects, inertial grade FOGs desire lower temperature dependence of EDF source than is presently available, although intrinsic thermal stability of EDF sources is one or two orders better than that of typical semiconductor sources. For inertial grade FOGs, it is desirable that the instability of the mean wavelength is suppressed below a few ppm against overall perturbation causes including both polarization and temperature. In addition, thermal effects could be minimized by optimum choice of operating parameters including the EDF length as well as the pump power [1].

In this paper, we report and experimentally demonstrate an optimum design of an EDF source with DPF/FRM configuration that has excellent stability of mean wavelength against temperature fluctuations as well as low polarization dependence. The temperature-dependent mean-wavelength change is found to be less than $1 \mathrm{ppm}$ while the source temperature changes by $\pm 5^{\circ} \mathrm{C}$, around its optimum operating temperature.

\section{SIMULATION}

For the numerical simulation of ASE propagation along the EDF in the DPF configuration, we followed 
the simulation method to solve the propagation equation in the previous literature [10]. In addition to general propagation behavior, thermal characteristics of ASE outputs were numerically analyzed. The propagation equation includes the inversion ratio as well as the absorption and the gain coefficients. The inversion ratio is also a function of the absorption and the gain coefficients which are all temperature dependent. Theoretical treatment of those parameters as a function of temperature is not easy and is beyond the scope of this paper. Instead, we tried to fit the experimentally obtained thermal data to a simple form of the absorption and the gain coefficients, which has been modeled by P. F. Wysocki [11].

The upper and lower energy manifolds of erbium transition responsible for $1550 \mathrm{~nm}$ radiation consist of 7 and 8 levels, respectively. Relative occupation probability of any two levels in each manifold in thermal equilibrium can be expected to follow a Boltzmann distribution as follows:

$$
\frac{P\left(E_{2}\right)}{P\left(E_{1}\right)}=\exp \left[-\frac{E_{2}-E_{1}}{k T}\right]
$$

where $E_{i}$ is the energy, $T$ is the absolute temperature, and $k$ is the Boltzmann constant. Based on the thermal statistics given in $\mathrm{Eq}$ (1), the following fitting forms were used to describe the absorption coefficient $(\alpha)$ and the gain coefficient $\left(\mathrm{g}^{*}\right)$ as a function of wavelength and temperature [11].

$$
\begin{aligned}
& \alpha(\lambda, T)=\alpha_{0}(\lambda) \exp \left[\frac{\beta_{a}(\lambda)}{k T}\right] \\
& g^{*}(\lambda, T)=g_{0}^{*}(\lambda) \exp \left[\frac{\beta_{e}(\lambda)}{k T}\right]
\end{aligned}
$$

The fitting parameters of $\alpha_{0}, g_{0}^{*}, \beta_{a}$, and $\beta_{e}$ can be determined from the measurement of the absorption coefficient and the gain coefficient as a function of wavelength at two different temperatures as in the following equations:

$$
\begin{aligned}
& \frac{\alpha\left(\lambda, T_{1}\right)}{\alpha\left(\lambda, T_{2}\right)}=\exp \left[\frac{\beta_{a}(\lambda)}{k}\left(\frac{1}{T_{1}}-\frac{1}{T_{2}}\right)\right] \\
& \frac{g^{*}\left(\lambda, T_{1}\right)}{g^{*}\left(\lambda, T_{2}\right)}=\exp \left[\frac{\beta_{e}(\lambda)}{k}\left(\frac{1}{T_{1}}-\frac{1}{T_{2}}\right)\right]
\end{aligned}
$$

In order to obtain the absorption/gain coefficients, it was assumed that the absorption/gain coefficients have the same spectral shapes as the absorption/emission cross sections that can be easily measured with a short length $(\sim 10 \mathrm{~cm})$ of EDF. First, we measured the spectral shapes of absorption/emission cross sections, and then the spectral peak value of absorption/gain coefficients were determined from the measurement of the absorption and the gain at a particular wavelength with a longer $(\sim 1 \mathrm{~m})$ EDF. The thermal parameters $\alpha_{0}, g_{0}^{*}, \beta_{a}$, and $\beta_{e}$ were determined from Eqs (3) by measurement of absorption/gain coefficients at two temperatures of $5{ }^{\circ} \mathrm{C}$ and $40^{\circ} \mathrm{C}$.

With the obtained absorption/gain coefficients as functions of wavelength and temperature, standard propagation equations [10] for the ASE and the pump were numerically solved with a set of boundary conditions given by the double-pass forward configuration. In the simulation, the EDF parameters such as core radius of $0.96 \mu \mathrm{m}$, Er-ion concentration of $2.65 \times 10^{19} \mathrm{~cm}^{-3}$, small signal absorption at $1.53 \mu \mathrm{m}$ of $17.1 \mathrm{~dB} / \mathrm{m}$ were used.

\section{EXPERIMENT}

The DPF/FRM source configuration with the measurement setup is shown in Fig. 1. A $1.48 \mu \mathrm{m}$ laser diode was used as a pump source. The pump light was launched into the EDF through a wavelength-division -multiplexed (WDM) fiber coupler. The pumped EDF produces ASE both in the forward (away from the pump) and backward (toward the pump) directions. The backward ASE is directed by the WDM coupler to a fiberpigtailed FRM. Owing to the FRM, the ASE signal double-passes the EDF experiencing orthogonal polarization states on each pass, which can reduce the effects of PDG. The details and advantages of the DPF/FRM configuration were described in [9]. The output isolator prevents time-dependent reflections from introducing undesirable fluctuation in the source mean wavelength.

The spectrum of the source output was measured by using an optical spectrum analyzer (OSA, Ando model AQ6317C). The mean wavelength of the source output was calculated from the measured output by using the formula as defined in $[5,10]$. A polarizer was used at the OSA input to simulate the polarizer in the gyro chip. In order to measure the thermal characteristics of the EDF source, a thermal chamber was used, where the inside temperature was controlled with $0.1^{\circ} \mathrm{C}$ resolution. Either the EDF only or the entire source (including other components as well as EDF) was put into the thermal chamber, depending on the experimental needs.

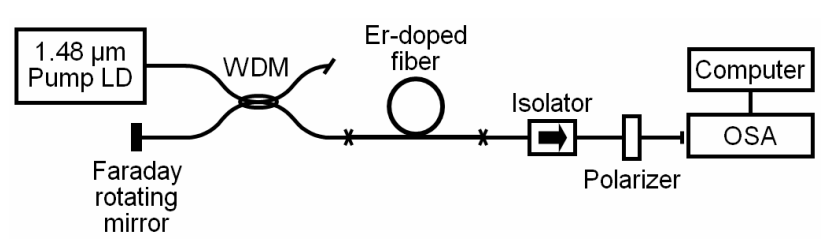

FIG. 1. Source configuration with the measurement setup. (OSA: optical spectrum analyzer) 


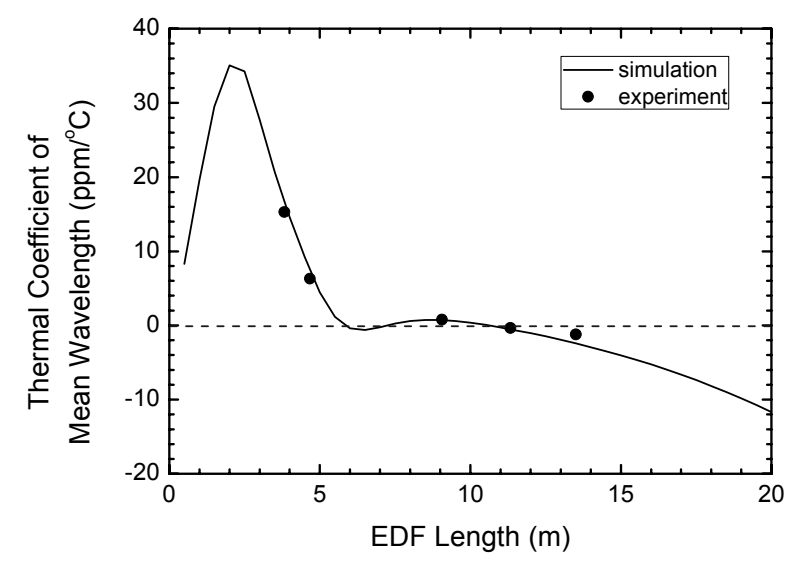

FIG. 2. Thermal coefficients of the mean wavelength at the temperature of $20^{\circ} \mathrm{C}$ and the pump power of $40 \mathrm{~mW}$. The solid line is the simulation and the dots are measured data.

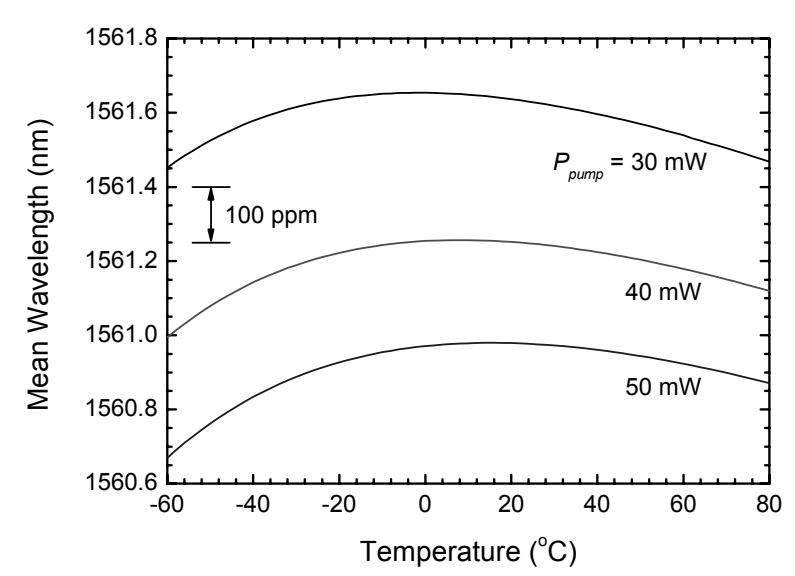

FIG. 3. Simulation results of mean wavelength vs. temperature at the EDF length of $11.3 \mathrm{~m}$ for various pump power.

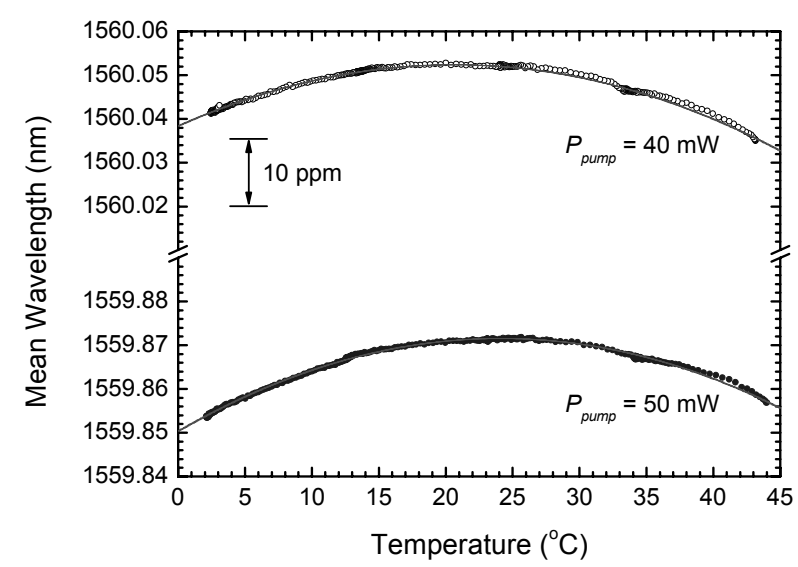

FIG. 4. Measured data of mean wavelength vs. temperature at the EDF length of $11.3 \mathrm{~m}$. Solid lines are fitted curves.

\section{RESULTS}

Figure 2 shows the simulation result of thermal coefficients of the mean wavelength, plotted with experimentally measured data for several EDF lengths. In the figure, the thermal ceoefficient is defined as $[\partial\langle\lambda\rangle / \partial T] /\langle\lambda\rangle$ in $\mathrm{ppm} /{ }^{\circ} \mathrm{C}$, where $\langle\lambda\rangle$ is the mean wavelength. It should be noted that the thermal coefficients are generally nonlinear in temperature, as will be described later. The numerical simulation and the measurement were made at the temperature of $20^{\circ} \mathrm{C}$ and at the pump power of $40 \mathrm{~mW}$. The simulation and the measurement are in good agreement, which verifies the validity of our simulations. The overall tendency of the curve is that it runs from positive coefficient to the negative one (except for the very short length region). Thus, we can see that at least one zero-crossing EDF length exists in this configuration. The zero thermal coefficient means that the mean wavelength is stable for small variation of the temperature. In Fig. 2, there exist several EDF lengths (around $6 \mathrm{~m}$ and $11 \mathrm{~m}$ ) that give zero thermal coefficient. Among these lengths, the longer length solution (around $11 \mathrm{~m}$ ) has an advantage in respect of polarization problem, since polarization effects that affect the meanwavelength stability can be more effectively suppressed with the longer EDF in the DPF/FRM configuration $[8,9]$. In addition, the output of the source with the longer EDF has nearly a Gaussian spectrum. Thus we closely examined the temperature dependence of the mean wavelength for the EDF length of $11 \mathrm{~m}$ or longer.

Figure 3 shows the simulation results of the mean wavelength vs. temperature for the EDF length of $11.3 \mathrm{~m}$. One can find that the zero-slope temperature exists near the room temperature at the pump power of $40 \mathrm{~mW}$ or $50 \mathrm{~mW}$. For lengths other than $11.3 \mathrm{~m}$, the zero-slope temperature shifts out of room temperature range or does not exist in the temperature range of our interest. (data not shown here)

The mean wavelength of the source was measured while varying the EDF (EDF only) temperature in the range from $2^{\circ} \mathrm{C}$ to $44^{\circ} \mathrm{C}$ and the results are shown in Fig. 4. The experimental results show nearly the same trend in the curve shape as the simulation, though there are some differences in absolute value of mean wavelength between them. Zero-slope temperatures also exist around the room temperature. These zero-slope temperatures can be tuned to some extent by varying the pump power. The measured data show that the mean wavelengths have their maxima at $19.5^{\circ} \mathrm{C}$ and $25.0^{\circ} \mathrm{C}$ for the pump power of $40 \mathrm{~mW}$ and $50 \mathrm{~mW}$, respectively. If the source is operated around the zero-slope temperature, the mean wavelength of the source becomes very stable against temperature fluctuations. For example, thermal variation of the mean wavelength could be less than $0.7 \mathrm{ppm}$ when the temperature range is within $20 \pm 5^{\circ} \mathrm{C}$, according to the fitted curve of the measured data at the pump 


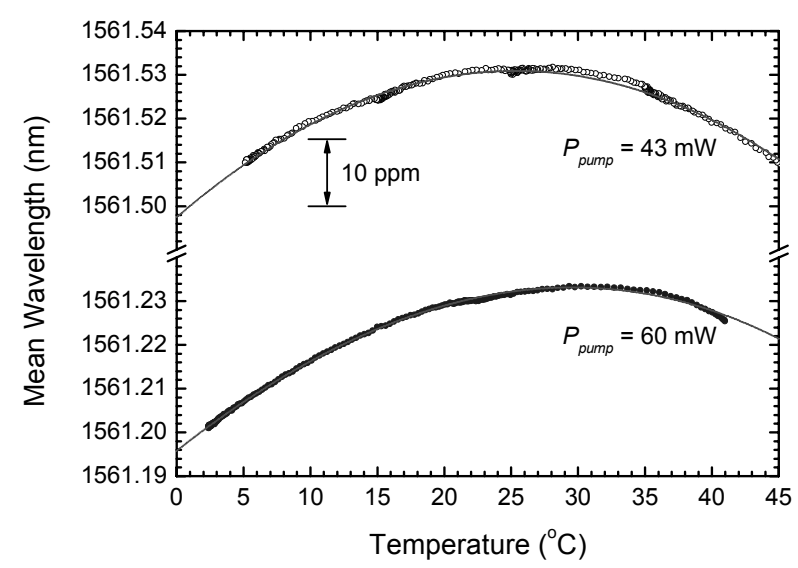

FIG. 5. Temperature dependence of the entire source (including WDM, isolator, and FRM as well as EDF) with the EDF length of $13.5 \mathrm{~m}$. Solid lines are fitted curves.

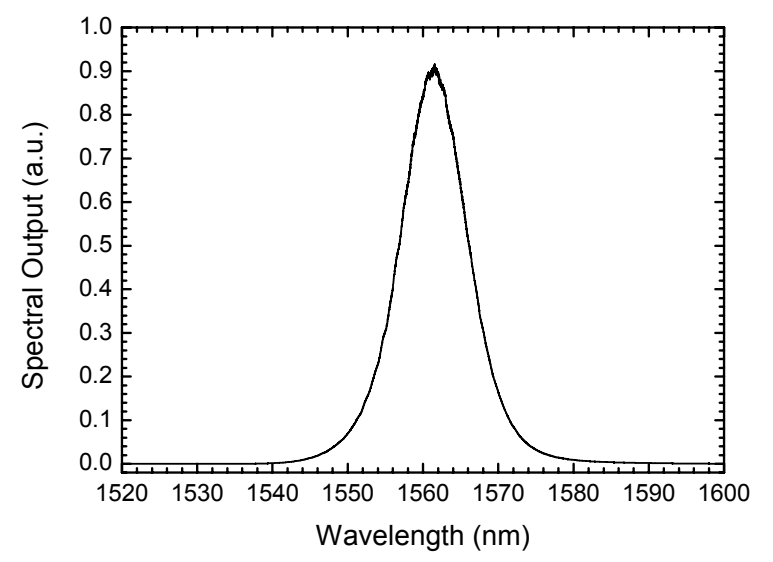

FIG. 6. Measured output spectrum of the source.

power of $40 \mathrm{~mW}$.

In the case of the actual source, not only the EDF but also the other components such as WDM, isolator and FRM affect the thermal behavior of the source mean wavelength. To see the temperature dependence of the entire source, the entire source including the EDF and the other components was put in the thermal chamber, and the source mean wavelength was measured while varying the chamber temperature. (The temperature change of the pump LD was maintained with $0.01^{\circ} \mathrm{C}$ with its own thermoelectric cooler.) The general trend of the mean wavelength vs. temperature was similar, but shifted to higher temperature compared with Fig. 4. The zero-slope for the stable mean wavelength was found at the higher temperature. In order to obtain stable mean wavelength near the room temperature, the longer EDF was needed. With the EDF length of $13.5 \mathrm{~m}$, the mean wavelength maxima occurred at room temperature as shown in Fig. 5. Figure 5 exhibits a parabolic nature similar to Fig. 4, except that the curvature differs a little due to the temperature effects of other components than the EDF, presumably the WDM. At the pump power of $43 \mathrm{~mW}$, optimum operating temperature (yielding maximum of mean wavelength) was $25.3^{\circ} \mathrm{C}$. With the temperature variation of $\pm 5^{\circ} \mathrm{C}$ and $\pm 10^{\circ} \mathrm{C}$ centered at $25^{\circ} \mathrm{C}$, the variations of the mean wavelength were 0.8 $\mathrm{ppm}$ and $3.3 \mathrm{ppm}$, respectively. With increasing the pump power, optimum operating temperature (zero-slope temperature) was also increased. When the pump power was increased to $60 \mathrm{~mW}$, optimum operating temperature was shifted to $29.8^{\circ} \mathrm{C}$. The optimum operating temperature was tunable with the pump power at the rate of $\sim 0.3^{\circ} \mathrm{C}$ $/ \mathrm{mW}$.

Finally, the measured spectrum of the source is shown in Fig. 6. The spectrum has a peak at $1561 \mathrm{~nm}$ with nearly Gaussian shape, which seems different from those of typical EDF sources. This is because the shorter wavelength peak $(\sim 1530 \mathrm{~nm})$ is more absorbed than the longer wavelength peak $(\sim 1560 \mathrm{~nm})$ in the relatively long length of EDF with the present DPF configuration.

\section{CONCLUSION}

A broadband EDF source with thermally stable mean wavelength is simulated and experimentally demonstrated. With the DPF/FRM configuration, the EDF length and the operating pump power are optimized to obtain the thermal variation of the mean wavelength less than $1 \mathrm{ppm}$ in the temperature range of $20 \pm 5^{\circ} \mathrm{C}$. This means that sub-ppm thermal stability can be obtained even with the temperature control as rough as $10^{\circ} \mathrm{C}$. The optimum operating temperature can be tuned with a proper choice of the EDF length and the pump power. Thus the mean wavelength can be reproducibly controlled and stabilized with sub-ppm accuracy, which is very important point in realizing a practical navigation-grade fiber-optic gyroscope.

\section{ACKNOWLEDGMENT}

This research was supported by research fund of Chonbuk National University in 2008.

\section{REFERENCES}

1. P. F. Wysocki, M. J. F. Digonnet, B. Y. Kim, and H. J. Shaw, "Characteristics of erbium-doped superfluorescent fiber sources for interferometric sensor applications," J. Lightwave Tech. 12, 550-567 (1994).

2. K. Iwatsuki, "Er-doped superfluorescent fiber laser pumped by $1.48 \mu \mathrm{m}$ laser diode," IEEE Photon. Technol. Lett. 2, 237-238 (1990).

3. H. G. Park, K. A. Lim, Y.-J. Chin, and B. Y. Kim, "Feedback effects in erbium-doped fiber amplifier/source 
for open-loop fiber-optic gyroscope," J. Lightwave Tech. 15, 1587-1593 (1997).

4. H. G. Park, Y.-J. Chin, and B. Y. Kim, "Gain-clamped fiber amplifier/source for open-loop fiber-optic gyroscope," Electron. Lett. 35, 167-168 (1999).

5. D. C. Hall, W. K. Burns, and R. P. Moller, "High-stability $\mathrm{Er}^{3+}$-doped superfluorescent fiber sources,” J. Lightwave Tech. 13, 1452-1460 (1995).

6. R. P. Moeller, W. K. Burns, and N. J. Frigo, "Open-loop output and the scale factor stability in a fiber-optic gyroscope,” J. Lightwave Tech. 7, 262-269 (1989).

7. V. J. Mazurczyk and J. L. Zyskind, "Polarization dependent gain in erbium doped-fiber amplifiers," IEEE Photon. Technol. Lett. 6, 616-618 (1994).

8. D. G. Falquier, M. J. F. Digonnet, and H. J. Shaw, "A polarization-stable Er-doped superfluorescent fiber source including a Faraday rotator mirror," IEEE Photon. Technol. Lett. 12, 1465-1467 (2000).

9. H. G. Park, M. J. F. Digonnet, and G. Kino, "Er-doped superfluorescent fiber source with a $\pm 0.5 \mathrm{ppm}$ long-term mean-wavelength stability," J. Lightwave Tech. 21, 34273433 (2003).

10. Y.-J. Jin, Y. S. Heo, T. J. Kim, and H. G. Park, "Characteristic of erbium-doped fiber sources with doublepass forward configuration for gyroscope application," Hankook Kwanghak Heoji(in Korean) 3, 460-465 (2003).

11. P. F. Wysocki, N. Conti, and D. Holcomb, "Simple modeling approach for the temperature dependence of the gain of Erbium-doped fiber amplifiers," Proc. SPIE 3847, 214223 (1999). 